\title{
Fabrication of a micro-structured surface based on interfacial convection for drag reduction
}

\author{
DOU ZhaoLiang ${ }^{1}$, WANG JiaDao ${ }^{1 *}$, YU Feng $^{2} \&$ CHEN DaRong ${ }^{1}$ \\ ${ }^{1}$ State Key Laboratory of Tribology, Tsinghua University, Beijing 100084, China; \\ ${ }^{2}$ China Ship Research and Development Academy, Beijing 100192, China
}

Received September 17, 2010; accepted November 9, 2010

\begin{abstract}
Based on interfacial convection in the presence of solvent evaporation, a novel method for the fabrication of a micro-structured surface is proposed to facilitate drag reduction. A mixture was coated on a substrate through a specially developed spray-painting system. Micron scale pits formed spontaneously in the coated surface because of interfacial convection and deformation driven by the gradient of the interfacial tension. Experimental results indicated that particles in the mixture played a crucial role in pit formation, and with a suitable selection of particle size and dosage, the characteristic parameters of the pitting could be controlled. The drag reduction experiments were first performed in a water tunnel, and the results showed that the micro-structured surface had a remarkable drag reduction performance over a great range of flow speeds.
\end{abstract}

micro-structured surface, particle, pit, interfacial convection, drag reduction

Citation: Dou Z L, Wang J D, Yu F, et al. Fabrication of a micro-structured surface based on interfacial convection for drag reduction. Chinese Sci Bull, 2011, 56: 626-632, doi: 10.1007/s11434-011-4378-2

The potential for drag reduction techniques to help tackle the sharply rising cost of fossil fuels by enhancing the performance of marine vessels has attracted significant research interest in recent times. Among the wide ranging techniques for drag reduction, surfaces with specially designed structures, such as lotus-like ultra-hydrophobic surfaces [1-3], shark skin-like micro-structured surfaces [4-14], and other bionic non-smooth surfaces imitating living organisms in the natural world [15], have been established through numerous experimental and numerical simulation studies. In these studies, however, designed surfaces are manufactured by traditional mechanical methods [4-10], such as milling, turning, rolling and embossing, or using riblet films [12-14]. The methods are generally limited to small areas, which are not viable for real applications.

In practice, an interfacial instability [16-19] in the coating film may lead the surface morphology to have an unexpected structure. Applications of interfacial convection are

*Corresponding author (email: jdwang@mail.tsinghua.edu.cn) numerous, especially in chemical engineering and material processing [20]. In recent years, interfacial convection in the presence of solvent evaporation has been the subject of increasing investigation because of its important role in thin-polymer-film engineering during the drying process [21]. A few applications of interfacial convection in surface micro-structure fabrication have been reported in the literature. Maruyama et al. [22] proposed a solvent-casting method for the preparation of 2-dimensional submicron scale structures. Srinivasarao et al. [23] reported a simple and robust method using evaporative cooling to fabricate ordered micro-porous polymer films.

In this study, based on interfacial convection theory, a micro-structured surface was fabricated through a spraypainting technique, and the formation mechanism was discussed. In the preliminary evaluation of the drag reduction property, samples were tested in a water tunnel, and a remarkable drag reduction performance was obtained. The proposed simple method may have promising potential in the fabrication of drag reducing micro-structured surfaces 
for use in engineering applications.

\section{Experiments and methods}

\subsection{Surface fabrication}

The paint used for spray-painting is a mixture of several components, which are listed in Table 1. After being dispersed adequately by a stirrer, the mixture is coated on a pretreated substrate through a specially developed spraypainting system, shown schematically in Figure 1. The system is feasible for both plane and cylindrical substrates. The inclination angle $\alpha$ is adjustable for the plane substrates. Spray-painting parameters, such as pressure, distance, coating thickness, temperature and humidity, can be readily adjusted, and the parameter values for spray-painting are listed in Table 2.

\subsection{Surface analysis methods}

The characteristic parameters of a coated surface are meas ured with a non-contact surface profiler MicroXAM ${ }^{\mathrm{TM}}$ from ADE Phase-Shift, and the measurement data are analyzed using the Scanning Probe Image Processor (SPIP). The Confocal Laser Scanning Microscope (CLSM) is also used for surface analysis.

To identify the influence of micro particles and gravity direction on surface fabrication, the coating samples are

Table 1 Components of the mixture for spray-painting

\begin{tabular}{cc}
\hline Components & Mass percentage (\%) \\
\hline Butyl acetate & 12 \\
Dimethylbenzene & 8 \\
Micro particles of metallic oxide & $2-6$ \\
Fluoroethylene vinyl ether (ester) copolymer resin & $62-66$ \\
Polydimethylsiloxane resin & 2 \\
Hexamethylene diisocyanate & 10 \\
\hline
\end{tabular}

Table 2 Parameters and values for spray-painting

\begin{tabular}{cc}
\hline Parameters & Values \\
\hline Muzzle caliber of spray gun $(\mathrm{mm})$ & $1.1-1.5$ \\
Spray-painting pressure $(\mathrm{MPa})$ & $0.4-0.6$ \\
Spray-painting distance $(\mathrm{mm})$ & $150-250$ \\
Coating thickness $(\mu \mathrm{m})$ & $50-100$ \\
Spray-painting temperature $\left({ }^{\circ} \mathrm{C}\right)$ & $15-25$ \\
Spray-painting humidity $(\%)$ & $<60$ \\
\hline
\end{tabular}

prepared by adding particles of different sizes and dosages to the mixture, and by adjusting the inclination angle $\alpha$ from 0 and $90^{\circ}$ to $180^{\circ}$, as shown in Table 3 .

To analyze the formation mechanism of micron scale pits, an infrared thermometer SC660 from FLIR is used to observe the temperature changes and material transfer in the liquid film.

\subsection{Drag reduction test of surface}

Preliminarily drag reduction tests to evaluate the drag reduction performance of the micro-structured surface, were performed in a high-speed water tunnel with a closed circulation system as shown in Figure 2(a). The pumped water in the tunnel first flowed through an anti-revolving section to prevent rotation of the flow stream, and vertical stream-wise flow disturbance was eliminated by a honeycomb. Guide vanes in the four bends were also used to restrain flow separation and improve uniformity. All the measures taken above were to ensure the quality of the flow in the test section.

The skin friction drag on samples was measured in the test section of the water tunnel, as shown in Figure 2(b). In the test section, the sample was supported by a central axle, and two small gaps (less than $0.3 \mathrm{~mm}$ ) were interspersed between the sample and its front and back accessories. With the aid of slip rings, the sample could glide along the central axle. When water stream flowed over the sample surface, the skin friction drag that the sample suffered was trans-

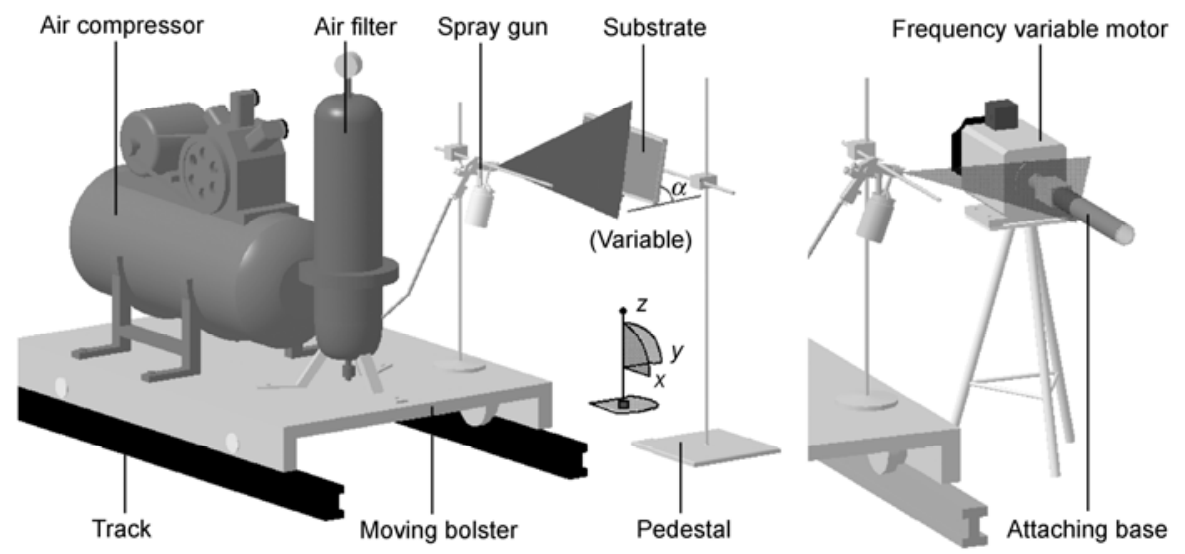

Figure 1 Schematic diagram of the spray-painting system. 
Table 3 Influencing factors during surface fabrication

\begin{tabular}{ccccc}
\hline Coatings & $\begin{array}{c}\text { Inclination } \\
\text { angle of } \\
\text { substrates } \alpha\end{array}$ & $\begin{array}{c}\text { Mean } \\
\text { diameter of } \\
\text { particles }(\mu \mathrm{m})\end{array}$ & $\begin{array}{c}\text { Mass } \\
\text { percentage of } \\
\text { particles }(\%)\end{array}$ & $\begin{array}{c}\text { Mass percent- } \\
\text { age of FEVE } \\
\text { resin }(\%)\end{array}$ \\
\hline FC15-2 & 0 & 14.41 & 2 & 66 \\
FC15-3 & 0 & 14.41 & 3 & 65 \\
FC15-4 & 0 & 14.41 & 4 & 64 \\
FC15-5 & 0 & 14.41 & 5 & 63 \\
FC15-6 & 0 & 14.41 & 6 & 62 \\
FC15-6-90 & $90^{\circ}$ & 14.41 & 6 & 62 \\
FC15-6-180 & $180^{\circ}$ & 14.41 & 6 & 62 \\
FC05-6 & 0 & 5.27 & 6 & 62 \\
FC30-6 & 0 & 29.59 & 6 & 62 \\
\hline
\end{tabular}

ferred to the force sensor by slip rings and pins, and the force signal was finally acquired by a computer for data analysis. As the fairing and tail of the model were fixed to the central support, the pressure force was transferred to the support, which means that the skin friction drag could be measured independently and directly in the test section of the water tunnel.

The test samples were cylindrical pipes $325 \mathrm{~mm}$ long and
$39 \mathrm{~mm}$ in diameter. Five samples were tested in the water tunnel. One sample was smooth with a surface roughness (RMS) of less than $1.6 \mu \mathrm{m}$, while other samples were painted with coatings FC05-6, FC15-2, FC15-6 and FC30-6. In the experiment, the flow speed of the water was 8-21 $\mathrm{m} / \mathrm{s}$, and the water temperature in the tunnel was $22.5^{\circ} \mathrm{C}$ (with fluctuations of less than $2^{\circ} \mathrm{C}$ ). The relative drag reduction ratio is defined here to evaluate the drag reduction performance, compared with the smooth sample:

$$
\lambda=\frac{F_{\mathrm{S}}-F_{\mathrm{C}}}{F_{\mathrm{S}}} \times 100 \%,
$$

where $\lambda$ is the drag reduction ratio of micro-structured surface, $F_{\mathrm{S}}$ is the friction drag of a smooth surface, and $F_{\mathrm{C}}$ is the friction drag of the micro-structured surface.

\section{Results and discussion}

\subsection{Surface analysis}

The characteristic parameters of the coated surface were

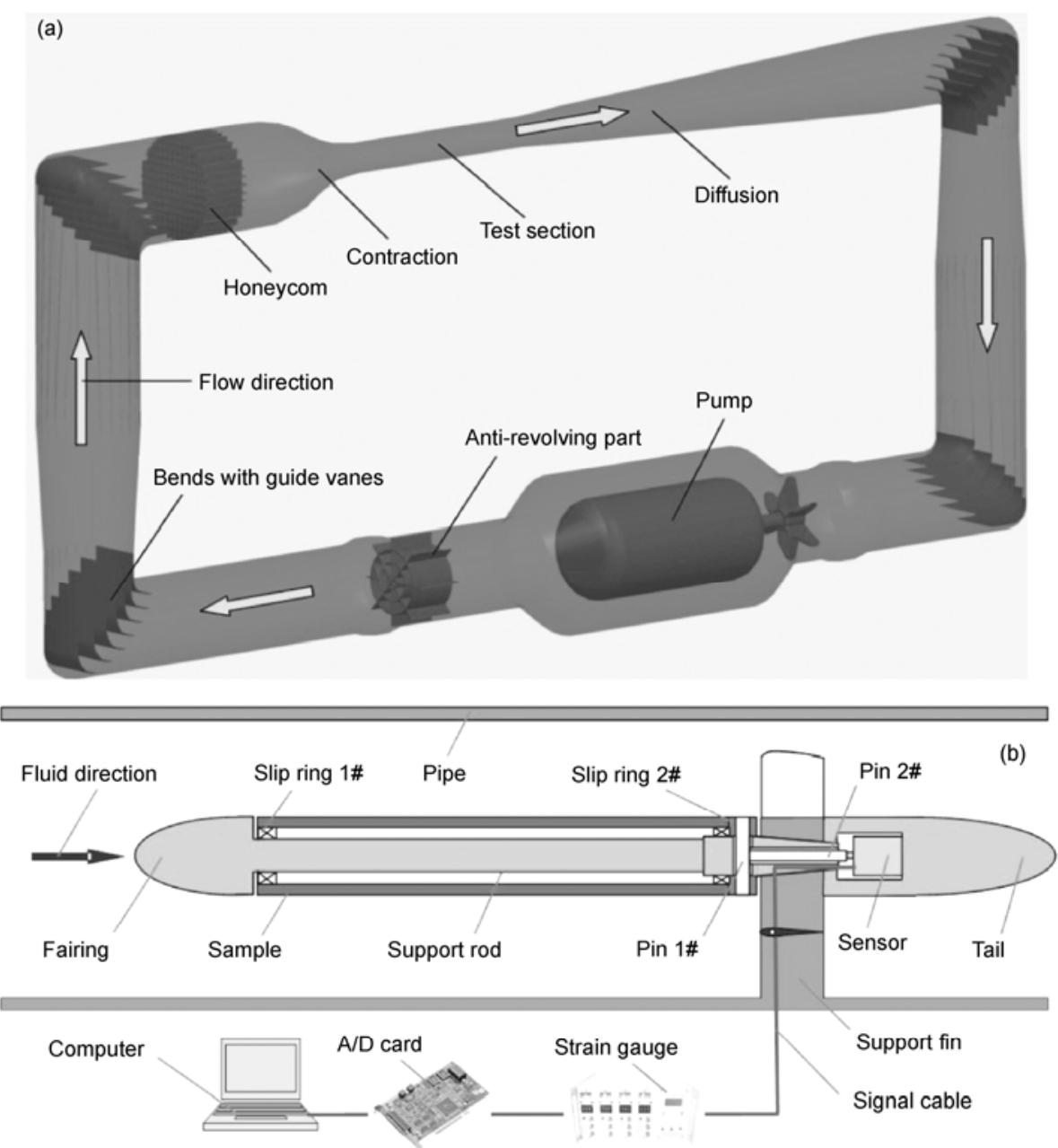

Figure 2 Schematic diagram of the high speed water tunnel for the skin friction drag test. (a) Flow circulation system; (b) test section. 
measured with a surface profiler MicroXAM ${ }^{\mathrm{TM}}$, and the measurement results are shown in Figure 3. Micron scale pits were distributed irregularly on the coated surface. The pits were shaped truncated cones with wider upsides and narrower downsides. As shown in Figure 4, the observed results of the CLSM indicated that, at the bottom of every pit, a particle existed, and the pits were similar in size and shape to the particles below. The corresponding relationship between pits and particles indicated that particles in the mixture may play an important role in pit formation.

To further clarify the influence of particles on pit formation, coating samples were fabricated by adjusting particle sizes and dosages in the mixture, and characteristic parameters of pits, such as depth, diameter, and area percentage were measured via SPIP. Here, the depth is a statistical distance from the datum plane to the bottom of the detected pits. The diameter is a statistical mean surface diameter counted at the detection level. The area percentage is defined as the ratio of the pit area to the total area of the detected
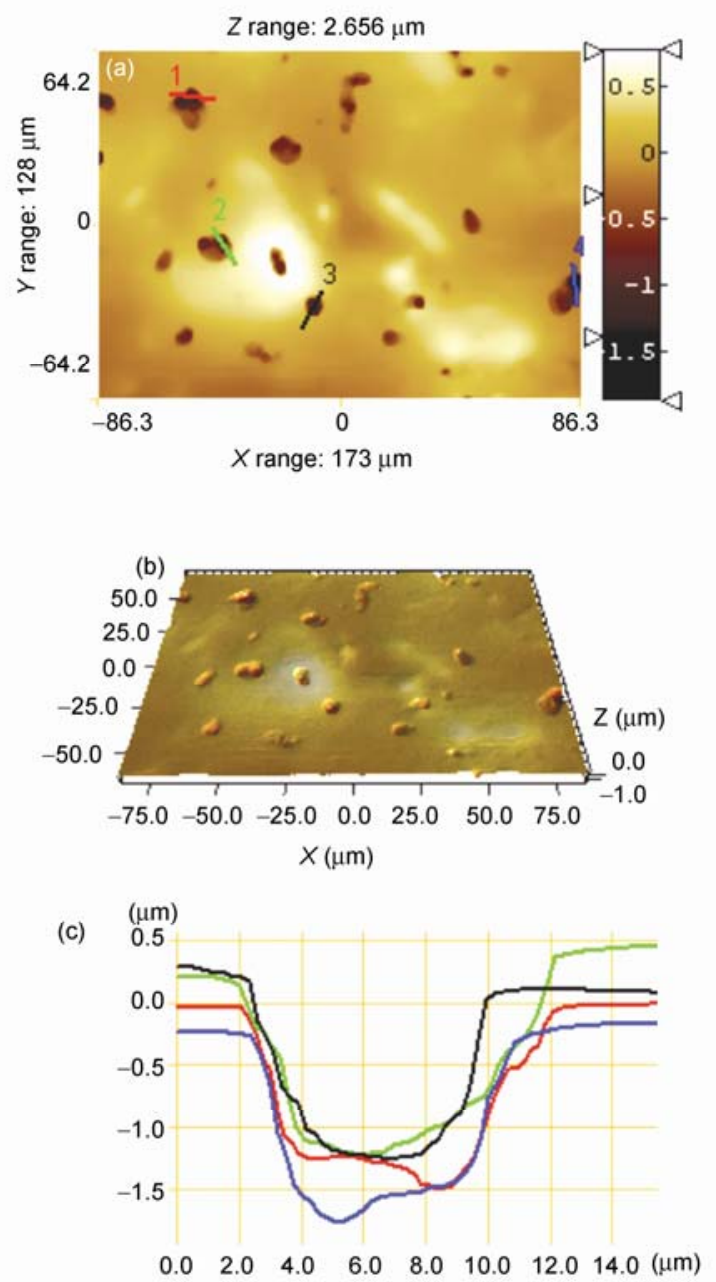

Figure 3 Micron scale pits distributed on the surface of coating FC15-3 (MicroXAM ${ }^{\mathrm{TM}}$ ). (a) 2-dimensional image of the micron pits with size and color of the segments corresponding to the size and depth of the pits; (b) 3-dimensional view of the pits; (c) profile curves of the pits.

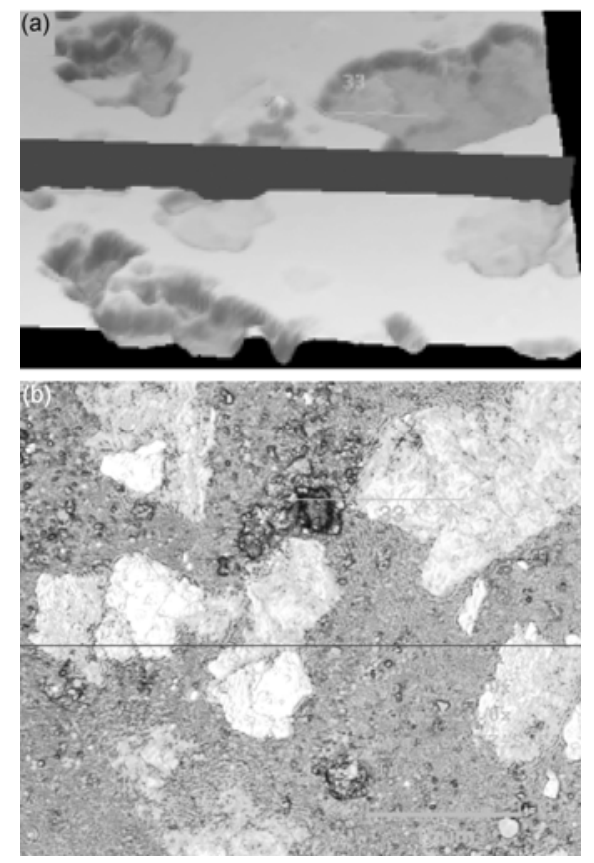

Figure 4 Micro-structured surface of coating FC15-6 under the CLSM. (a) 3-dimensional image of the micron scale pits in the coating surface; (b) optical micrograph of the subsurface layer where brighter areas represent particles.

surface. For all parameters, counts are based on the mean of nine counts, and three detected areas from three samples.

The relationship between the characteristic parameters of pits and particle sizes and dosages is shown in Figure 5. It can be seen from Figures 5(a) and (b) that, pit size, i.e. depth and diameter, increased with the increment of particle diameter from 5.27 to $29.59 \mu \mathrm{m}$, but was not influenced by particle dosage. As shown in Figure 5(c), the percentage area of pits increased when the particle dosage increased from $2 \%$ to $5 \%$, but was invariable with changes in particle size. Thus, pit size is mainly determined by particle size, and is independent of particle dosage. The percentage area of pits is related to particle dosage, and is independent of particle size. Therefore, with a suitable selection of particle size and dosage, size and area percentage of micron pits can be effectively controlled.

\subsection{Formation mechanism analysis of micro-structured surface}

Figure 6 shows the temperature change in the wet film, which was measured by an infrared thermometer SC660. The average temperature of the surface layer was $22^{\circ} \mathrm{C}$, while that of the bottom was $23.7^{\circ} \mathrm{C}$. In other words, there was a temperature decrease of $1.7^{\circ} \mathrm{C}$. Surface regions close to the particles had a higher temperature of $22.8^{\circ} \mathrm{C}$, while regions far from the particles had a lower temperature of $22^{\circ} \mathrm{C}$, which represented a visible temperature difference in the surface layer.

The above analysis indicated that solvent evaporation in 

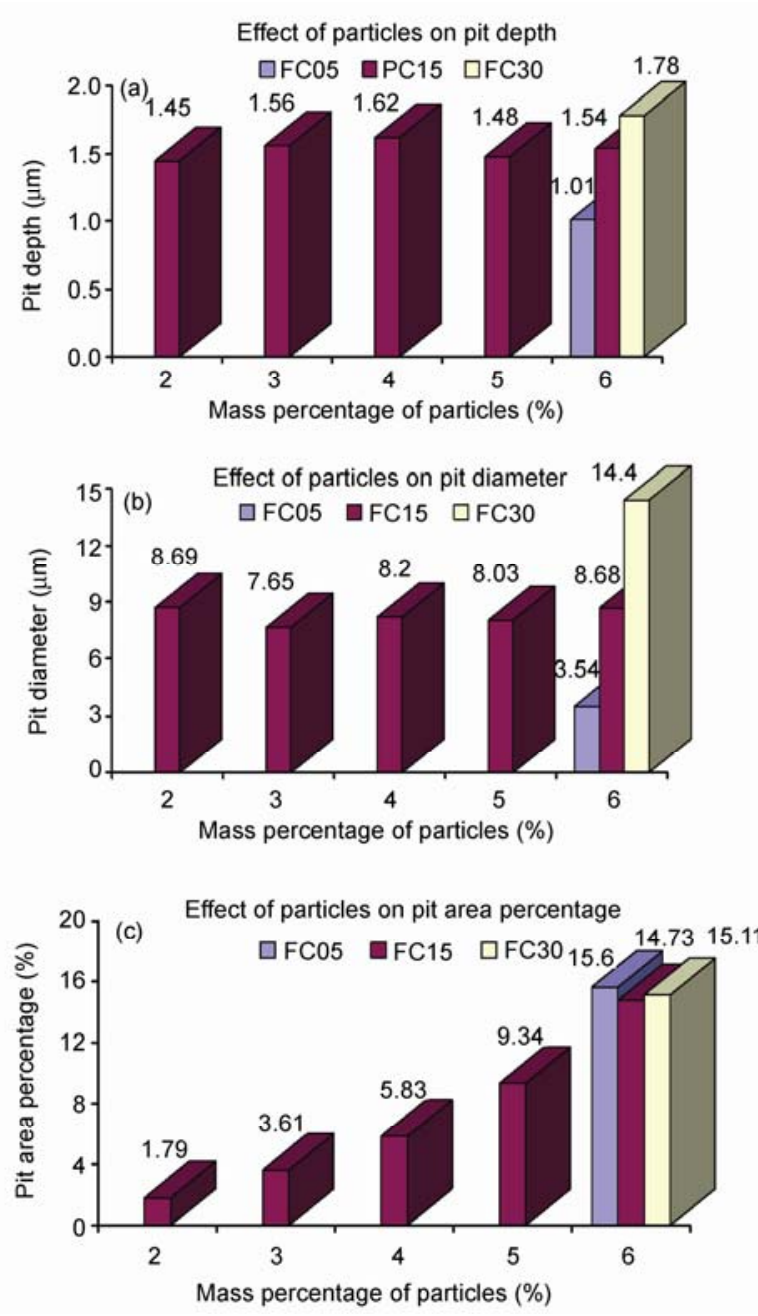

Figure 5 Effect of particle dosage and size on area percentage and size of pits.

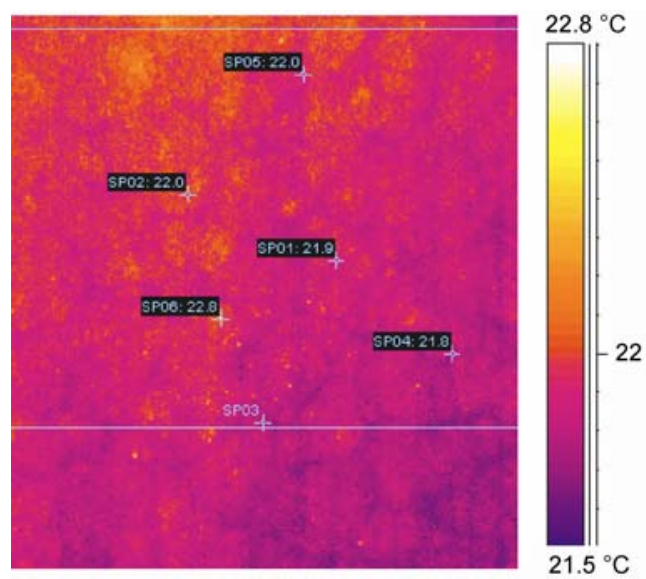

Figure 6 Infrared thermography of surface layer.

the wet film resulted in a temperature decrease and temperature difference at the surface layer. Regions with a higher temperature had lower interfacial tension, while regions with a lower temperature had higher interfacial ten- sion. Therefore, the temperature gradient created an interfacial tension gradient, and the interfacial tension induced interfacial convection [16-19] at the surface layer.

A sketch map of the formation mechanism of a microstructured surface is shown in Figure 7. The mixture at regions close to the particles with lower interfacial tension was driven to regions far from particles where the interfacial tension was higher. The material transfer from regions close to the particles led to subsidence compared with surrounding regions. The process was synchronous with coating solidification and viscosity increment. When the mixture became viscous enough, interfacial convection ceased, and interfacial deformation caused by convection became fixed, which resulted in micron scale pits in the coating surface.

Interfacial convection might, in fact, be driven by thermocapillary action due to the temperature dependent nature of the interfacial tensions or by the buoyancy arising from density differences. The relative size of these two effects depended on the fluid layer depth, with thermocapillary forces dominating in sufficiently thin layers [24].

To evaluate the relative importance of buoyancy and thermocapillary action in interfacial convection, coatings with different inclination angles were prepared in order to change the buoyancy direction (gravity direction), and the surface characteristic parameters were measured, as shown in Table 4.

From Table 4, characteristic parameters, such as depth,

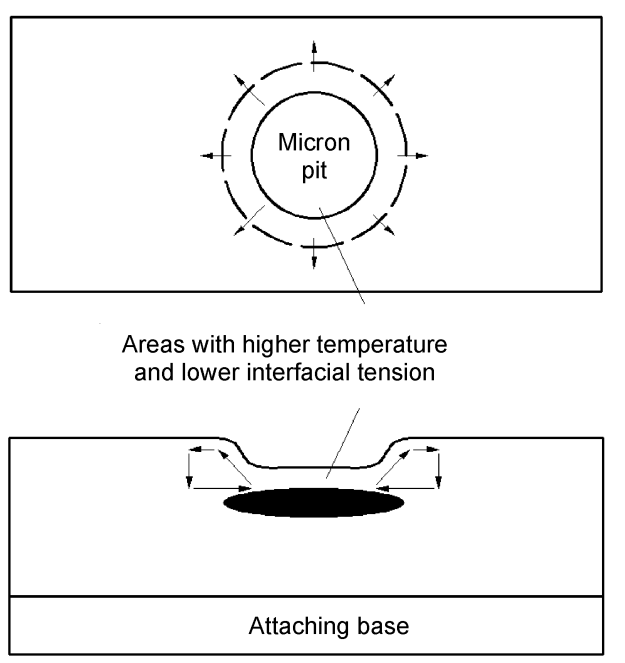

Figure 7 Sketch map of micro-structured surface formation.

Table 4 Influence of inclination angles on surface microtopography of coating FC15-6

\begin{tabular}{cccc}
\hline $\begin{array}{c}\text { Inclination } \\
\text { angles }\end{array}$ & $\begin{array}{c}\text { Depth of pits } \\
(\mu \mathrm{m})\end{array}$ & $\begin{array}{c}\text { Diameter of pits } \\
(\mu \mathrm{m})\end{array}$ & $\begin{array}{c}\text { Area percentage of } \\
\text { pits }(\%)\end{array}$ \\
\hline$\alpha=0^{\circ}$ & 1.54 & 8.68 & 14.73 \\
$\alpha=90^{\circ}$ & 1.49 & 8.76 & 14.59 \\
$\alpha=180^{\circ}$ & 1.57 & 8.56 & 14.91 \\
\hline
\end{tabular}


diameter and area percentage, show small differences. Accordingly, the change of buoyancy direction has little influence on surface fabrication. In other words, compared with thermocapillary action, the effect of buoyancy was negligible during interfacial convection. Therefore, interfacial convection and deformation driven by the interface tension gradient were main factors in micro-structured surface formation.

\subsection{Drag reduction performance of micro-structured surface}

Four coating samples were tested in the water tunnel to evaluate the drag reduction performance, and the test results are shown in Figure 8. From the figure, the drag reduction ratio for all tested samples was over $10 \%$ in a flow speed ranging from 8 to $21 \mathrm{~m} / \mathrm{s}$. With the increase in flow speed, the drag reduction performance became more remarkable. As the flow speed increased to $21 \mathrm{~m} / \mathrm{s}$, the average drag reduction ratio increased to $23.7 \%$, with a highest value of 28.4\% for FC15-6. Accordingly, a micro-structured surface reduces skin force effectively, and has remarkable drag reduction performance over a wide range of flow speeds.

It can also be seen from the figure that the drag reduction performances of the four coating samples were different because of the surface characteristic parameters. The variance between FC15-3 and FC15-6 indicates that the area percentage of the micro scale pits has a certain influence on drag reduction, while the difference between FC05-6, FC15-6 and FC30-6 shows that pit size also plays a role in drag reduction. Therefore, with the aim of maximizing the drag reduction efficiency, systemic experiments should be carried out to clarify the relationship between drag reduction performance and surface characteristic parameters, to optimize the parameters.

\section{Conclusions}

From the above experimental results and discussion, the following conclusions are drawn.

(1) Micro-structured surfaces formed spontaneously after the solidification of the spray-painted coating. Thus particles in the mixture play a key role in surface formation. With a suitable selection of particles, the characteristic parameters of micro-structured surface can be controlled.

(2) Solvent evaporation in the wet film induces a temperature gradient and interfacial tension gradient in the surface layer, and results in interfacial convection and deformation, which are considered to be the main factors in surface formation.

(3) Comparison experiments between smooth and microstructured surfaces were performed in a high speed water tunnel. Preliminary test results indicate that the micro-structured surface fabricated by the proposed method has a

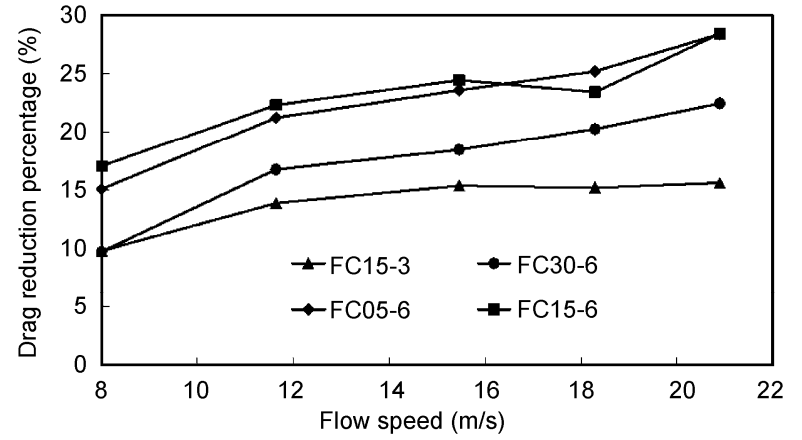

Figure 8 Drag reduction percentage of the micro-structured surface under different flow speeds.

remarkable drag reduction performance over a wide range of flow speeds, and the characteristic parameters have considerable influence on drag reduction efficiency.

In subsequent work, related research such as accurate control and optimization of surface characteristic parameters will be carried out to maximize drag reduction efficiency. Furthermore, drag reduction in the fields of aeronautical engineering such as air vehicles has also been the subject of increasing attention because of the significant potential practical value to both military and civil applications. Because the drag reduction tests in this paper were only performed in water flow, and a distinct change in the physical properties of fluid media may lead to unexpected results in the drag reduction performance of micro-structured surfaces, further investigation will be done on the feasibility and viability of micro-structured surface applications in air flow for drag reduction.

This work was supported by the National Natural Science Foundation of China (50721004, 50675112).

1 Wang J D, Yu Y, Chen D R. Research progress on the ultrahydrophobic surface topography effect. Chinese Sci Bull, 2006, 51: 2297-2300

2 Li J, Zhou M, Cai L, et al. On the measurement of slip length for liquid flow over super-hydrophobic surface. Chinese Sci Bull, 2009, 54: 4560-4565

3 Lu S, Yao Z H, Hao P F, et al. Drag reduction in ultrahydrophobic channels with micro-nano structured surfaces. Sci China-Phys Mech Astron, 2010, 53: 1298-1305

4 Walsh M J. Turbulent boundary layer drag reduction using riblets. AIAA Paper, 1982, 82-0169

5 Walsh M J. Riblets as a viscous drag reduction technique. AIAA J, 1983, 21: 485-486

6 Suzuki Y, Kasagi N. Turbulent drag reduction mechanism above a riblet surface. AIAA J, 1994, 32: 1781-1790

7 Bechert D W, Bruse M, Hage W, et al. Experiments on drag-reducing surfaces and their optimization with an adjustable geometry. J Fluid Mech, 1997, 338: 59-87

8 Bechert D W, Bruse M, Hage W. Experiments with three-dimensional riblets as an idealized model of shark skin. Exp Fuilds, 2000, 28: 403-412

9 Zhou G. Research on drag reduction by traveling wave and development of mini-sized high-speed water tunnel. Doctoral Dis- 
sertation. Beijing: Tsinghua University, 2008

10 Han X, Zhang D Y, Li X, et al. Bio-replicated forming of the biomimetic drag-reducing surfaces in large area based on shark skin. Chinese Sci Bull, 2008, 53: 1587-1592

11 Du Y Q, Symeonidis V, Karniadakis G E. Drag reduction in wallbounded turbulence via a transverse traveling wave. J Fluid Mech, 2002, 457: 1-34

12 Sundaram S, Viswanath P R, Rudrakumar S. Viscous drag reduction using riblets on NACA 0012 airfoil to moderate incidence. AIAA J, 1996, 34: 676-682

13 McLean J D, George F D N, Sullivan P P. Flight-test of turbulent skin-friction reduction by riblets. RAES, 1987, 408-428

14 Li Y B, Qiao Z D. An experimental research of drag reduction using riblets for the Y-7 airplane. Aerodyn Exp Measure Control, 1995, 9: 24-26

15 Ren L Q. Progress in the bionic study on anti-adhesion and resistance reduction of terrain machines. Sci China Ser E-Tech Sci, 2009, 52: 273-284

16 Borciaa R, Bestehorn M. Phase-field simulations for evaporation with convection in liquid-vapor systems. Eur Phys J B, 2005, 44: 101-108

17 Souche M, Clarke N. Interfacial instability in bilayer films due to solvent evaporation. Eur Phys J E, 2009, 28: 47-55

18 Ozen O, Johnson D, Narayanan R. Observations on interfacial convection in multiple layers without and with evaporation. Lect Notes Phys, 2003, 628: 59-77

19 Heriot S Y, Jones R A L. An interfacial instability in a transient wetting layer leads to lateral phase separation in thin spin-cast polymer-blend films. Nat Mater, 2005, 4: 782-786

20 Johnson D, Narayanan R. Marangoni convection in multiple bounded fluid layers and its application to materials processing. Philos Trans, 1998, 56: 885

21 Jukes P C, Heriot S Y, Sharp J S, et al. Time-resolved light scattering studies of phase separation in thin film semiconducting polymer blends during spin-coating. Macromolecules, 2005, 38: 2030

22 Maruyama N, Koito T, Sawadaishi T, et al. Mesoscopic pattern formation of nanostructured polymer assemblies. Supramol Sci, 1998, 5: $331-336$

23 Srinivasarao M, Ollings D, Philips A, et al. Three-dimensionally ordered array of air bubbles in a polymer film. Science, 2001, 292: 79-83

24 Tavener S J, Cliffe K A. Two-fluid marangoni-Benard convection with a deformable interface. J Comput Phys, 2002, 182: 277-300

Open Access This article is distributed under the terms of the Creative Commons Attribution License which permits any use, distribution, and reproduction in any medium, provided the original author(s) and source are credited. 